\title{
Co-expression of FOXL1 and PP2A inhibits proliferation inducing apoptosis in pancreatic cancer cells via promoting TRAIL and reducing phosphorylated MYC
}

\author{
YUNWEI LI, DONGYANG YU, WEIWEI SHENG, HE SONG, YUJI LI and MING DONG \\ Department of General Surgery, Gastrointestinal Surgery, The First Hospital of China \\ Medical University, Shengyang 110001, Liaoning, P.R. China
}

Received July 13, 2015; Accepted September 17, 2015

DOI: $10.3892 /$ or.2016.4592

\begin{abstract}
Pancreatic cancer is usually diagnosed in the advanced stages and is sensitive to only few therapies. The forkhead box L1 (FOXL1) and protein phosphatase 2A (PP2A) have been recognized to be tumor suppressive in human pancreatic cancers. In the present study, we co-expressed the two tumor suppressive molecules with a '2A peptide' linker, which guaranteed the two molecules were transcribed into one mRNA, whereas they were translated into two separate proteins, in pancreatic cancer Panc-1 cells, and investigated the inhibition of the two molecules on the proliferation and migration of Panc-1 cells. Results demonstrated that, either overexpression of FOXL1 or PP2A via adenovirus significantly inhibited the proliferation of Panc-1 cells, whereas promoted apoptosis in such cells. Moreover, the co-expression of both FOXL1 and PP2A exerted synergistic antitumor effect in Panc-1 cells, with significantly higher proliferation inhibition and tumor induction. In addition, we found that the overexpressed FOXL1 promoted the TNF-related apoptosis-inducing ligand (TRAIL), whereas the overexpressed PP2A downregulated the phosphorylation of c-MYC. The co-expression of FOXL1 and PP2A presented both functions in Panc-1 cells. In conclusion, the adenovirus-mediated co-expression of FOXL1 and PP2A with the 2A peptide linker exterts synergistic suppression of pancreatic cancer cells via inhibiting the growth and promoting apoptosis of cancer cells, probably via upregulating TRAIL and reducing the phosphorylation of MYC.
\end{abstract}

\section{Introduction}

Pancreatic cancer, mainly pancreatic ductal adeno carcinoma (PDAC), is one of the most deadly and aggressive

Correspondence to: Dr Ming Dong, The First Hospital of China Medical University, 155 Nanjingbei Street, Heping, Shengyang 110001, P.R. China

E-mail: cmudongming@sohu.com

Key words: pancreatic cancer, FOXL1, PP2A, proliferation and migration, TRAIL, MYC cancers (1). It is the world-wide seventh (2), and the fourth in the United States (3) of most common cause of cancer-related death, with a poor prognosis and swift progression before death. Surgical eradication is still the only potentially curative treatment of this malignancy, but there are only $15-20 \%$ cases indicative for surgery, because of the early occurrence of local advancement or distal metastasis (4). Five-year overall survival rate ranges from 1 to $6 \%(3,5,6)$. Even after surgical eradication plus adjuvant chemotherapy, OS rates do not exceed $30 \%(5,6)$.

Various chemotherapic agents have failed to improve survival of pancreatic cancer patients. In recent years, FOLFIRINOX, a cocktail of 5-fluorouracil (5'-FU), irinotecan and oxaliplatin has significantly improved OS of pancreatic patients with metastasis, compared with the singe treatment with gemcitabine $(7,8)$. Besides conventional chemotherapy, accumulating understanding of the biological pathogenesis of pancreatic cancer has provided a variety of targeted approaches. However, except erlotinib, which is an inhibitor to epidermal growth factor receptor (EGFR), no other targeted therapy has as yet demonstrated significant effect against pancreatic cancer $(9,10)$. Insulin-like growth factor 1 (IGF-1) and its receptor, PI3-kinase/Akt/mTOR and mitogen-activated protein kinases/extracellular signal-regulated kinases (MEK/ERK) pathway are upregulated in the majority of PDACs $(11,12)$. However, a phase II trial on a monoclonal antibody against IGF receptor (IGFR) indicates no significant effect in OS and progression-free survival (PFS) for PDAC patients (4). The chemical agents targeting the PADC-overexpressed vascular endothelial growth factor (VEGF) which promotes cancer angiogenesis and metastasis (13), also failed to improve PFS and OS (14). Other treatments targeting farnesyltransferase, the tumor stroma in pancreatic cancer or autophagy are on the way.

Forkhead box L1 (FOXL1) belongs to a forkhead/winged helix-box (FOX) family of transcription factors. All Fox members, being classified as FOXA to FOXR (15), Fox molecules play critical roles in a variety of physiologic (16) or pathologic processes such as cancer, as tumor suppressors (17-19). Particularly, FOXM1 was identified to be oncogenic in pancreatic cancer, and is associated with poor prognosis and pathologic stage of PADC $(20,21)$, whereas, FOXL1 has 
recently been recognized as a tumor suppressor in PADC (22). Therefore, FOXL1 might be another target for the treatment of pancreatic cancers. Protein phosphatase 2A (PP2A) is a large collection of oligomeric protein serine/threonine phosphatases and accounts for a large fraction of phosphatase activity in eukaryotic cells. PP2A is a critical tumor suppressor, via controlling a number of cellular processes, including cell cycle progression $(23,24)$. Key signaling pathways that are negatively regulated by PP2A include members of the MAPK/ERK pathways, NF- $\kappa \mathrm{B}$, and c-Myc signaling (25). In particular, PP2A suppresses the oncogenic activity (26) of c-Myc via specifically dephosphorylating the key serine 62 (S62) in c-Myc (27), stimulating its ubiquitination (28) and thus accelerating the degradation of c-Myc. The overexpression of endogenous PP2A inhibitors, such as SET (I2PP2A) and cancerous inhibitor of PP2A (CIP2A) in head and neck squamous cell carcinoma, colon cancer, gastric cancer, breast cancer, and most recently, pancreatic cancer $(29,30)$. Thus, the tumor suppressive PP2A might also facilitate pancreatic cancer therapy.

In the present study, we constructed a recombinant adenovirus, which carries the coding sequence both of FOXL1 and PP2A, with a self-cleavage sequence. Then we evaluated the regulation of the recombinant adenovirus on the proliferation of pancreatic cancer cells, on the sensitivity of pancreatic cancer cells to 5'-FU. In addition, we investigated the activation of TNF-related apoptosis-inducing ligand (TRAIL) by the recombinant virus. The present study provides a novel antitumor strategy against PADC.

\section{Materials and methods}

Cell lines and culture conditions. Human pancreatic carcinoma Panc-1 cells were purchased from American Type Culture Collection (ATCC, Manassas, VA, USA). Cells were cultured in RPMI-1640 media (Gibco Life Technologies, Rockville, MD, USA) supplemented with GlutaMAX-I (Invitrogen, Carlsbad, CA, USA), $50 \mathrm{IU} / \mathrm{m}$ penicillin and $50 \mathrm{mg} / \mathrm{ml}$ streptomycin (both from CSPC Zhongnuo Pharmaceutical Co., Ltd., Shijiazhuang, China) and 10\% (v/v) fetal bovine serum (FBS) (Sijiqing, Hangzhou, China). Cells were incubated at $37^{\circ} \mathrm{C}$ in a humidified incubator with $5 \% \mathrm{CO}_{2}$. For the treatment with $10 \mu \mathrm{M}$ 5'-FU (Sigma-Aldrich, St. Louis, MO, USA), cells at $>85 \%$ confluence were inoculated with RPMI-1640 media supplemented with $2 \%$ FBS, containing $10 \mu \mathrm{M}$ 5'-FU.

Adenovirus-mediated coexpression of FOXL1 and PP2A. Human FOXL1 and PP2A coding sequences (both from Sinobio, Beijing, China) were amplified, respectively, or were overlapped with with $2 \mathrm{~A}$ peptide coding sequence as a linker. Then each coding sequence of FOXL1 or PP2A, the over-lapped FOXL1-2A-PP2A was cloned into the the pShuttle-CMV vector to generate the recombinant plasmid of pShuttle-CMV-FOXL1, pShuttle-CMV-PP2A, or pShuttle-CMV-FOXL1-2A-PP2A, the control pShuttle-CMV-con was generated with an insertion of the coding sequence of enhanced green fluorescence protein (EGFP). The recombinant adenovirus, Ad (FOXL1), Ad (PP2A), Ad (FOXL1 + PP2A) or the Ad (con) virus was rescued under the guidance of the vector manual. To overexpress FOXL1, PP2A, or both molecules in Panc-1 cells,
Panc-1 cells were infected with the Ad (FOXL1), Ad (PP2A), Ad (FOXL1 + PP2A) or the Ad (con) virus with 1 or 3 multiplicities of infection (MOI) for $2 \mathrm{~h}$ at $37^{\circ} \mathrm{C}$, and then were updated with fresh RPMI-1640 media supplemented with $2 \%$ FBS.

Quantitative assay for the mRNA level of FOXL1 or PP2A. Total cellular mRNA in Panc-1 cells was isolated with TRIzol reagent (Life Technologies, Grand Island, NY, USA) and was supplemented with RNase inhibitor (Takara, Tokyo, Japan). mRNA samples were directly quantified via real-time PCR, using the SuperScript III Platinum One-Step qRT-PCR kit (Qiagen GmbH, Hilden, Germany) on an ABI PRISM 7300 detection system. The housekeeping $\beta$-actin gene was simultaneously quantified to standardize the amount of target mRNA. Relative quantification of gene transcription level was performed by the $-\Delta \Delta C t$ method (31), the relative target mRNA was presented as relative level to the control group.

Western blot assay. Harvested Panc-1 cells post various treatment were promptly homogenized in a Cell Lysis Buffer (Cell Signaling Technology Inc., Danvers, MA, USA), then centrifuged at $13,000 \mathrm{x}$ g for $20 \mathrm{~min}$ at $4^{\circ} \mathrm{C}$ to collect the supernatant. Next, each sample was quantified with a BCA protein assay reagent kit (Pierce, Rockford, IL, USA) and was diluted to same concentration. Before being added with loading buffer and being boiled, protein samples with equal amount were separated with $10 \%$ sodium dodecyl sulfate-polyacrylamide (SDS-PAGE) electrophoresis and then were transferred to a PVDF membrane (Millipore, Bedford, MA, USA). After the block with $2 \%$ bovine serum albumin (BSA) (Ameresco, Framingham, MA, USA) overnight at $4^{\circ} \mathrm{C}$, the membrane was incubated overnight again at $4^{\circ} \mathrm{C}$ with the rabbit polyclone antibody against FOXL1, PP2A, $\beta$-actin, caspase-3, poly(ADP-ribose) polymerase (PARP), TRAIL, MYC or phosphorylated MYC (Ser at 62). After triple washes with Tris-buffered saline and Tween-20 (TBST), the membrane was incubated with horseradish peroxidase-linked secondary anti-rabbit antibody (Sigma-Aldrich) for an inoculation for $1 \mathrm{~h}$ at room temperature. The specific bingding was scanned via a molecular dynamics densitometer (Imaging Technology, Ontario, Canada). ImageJ software was used to quantify band density.

Growth curve assay and colony formation assay. The proliferation of Panc-1 cells was evaluated via cell counting assay, briefly as follows. Panc-1 cells were quantitatively seeded in 12 -well plates with $10^{4} / \mathrm{ml}$, post an inoculation for $8 \mathrm{~h}$ (cells closely attached), cells were infected with $3 \mathrm{MOI}$ of Ad (FOXL1), Ad (PP2A), Ad (FOXL1 + PP2A) or Ad (con) respectively, and were incubated at $37^{\circ} \mathrm{C}$ for $1,3,5$ or 7 days. Then cells in each well were trypsinized and were counted in a hemocytometer with the use of trypan blue staining. Colony formation assay was also utilized to determine the proliferation of Panc-1 cells, 1,000 cells were seeded into a 6-well plate, and were infected with $3 \mathrm{MOI}$ of Ad (FOXL1), Ad (PP2A), Ad (FOXL1 + PP2A) or Ad (con), respectively; then cells were incubated at $37^{\circ} \mathrm{C}$ for another 5 days, and the cell colonies were stained by $0.5 \%$ crystal violet (Sigma-Aldrich) and were counted. 

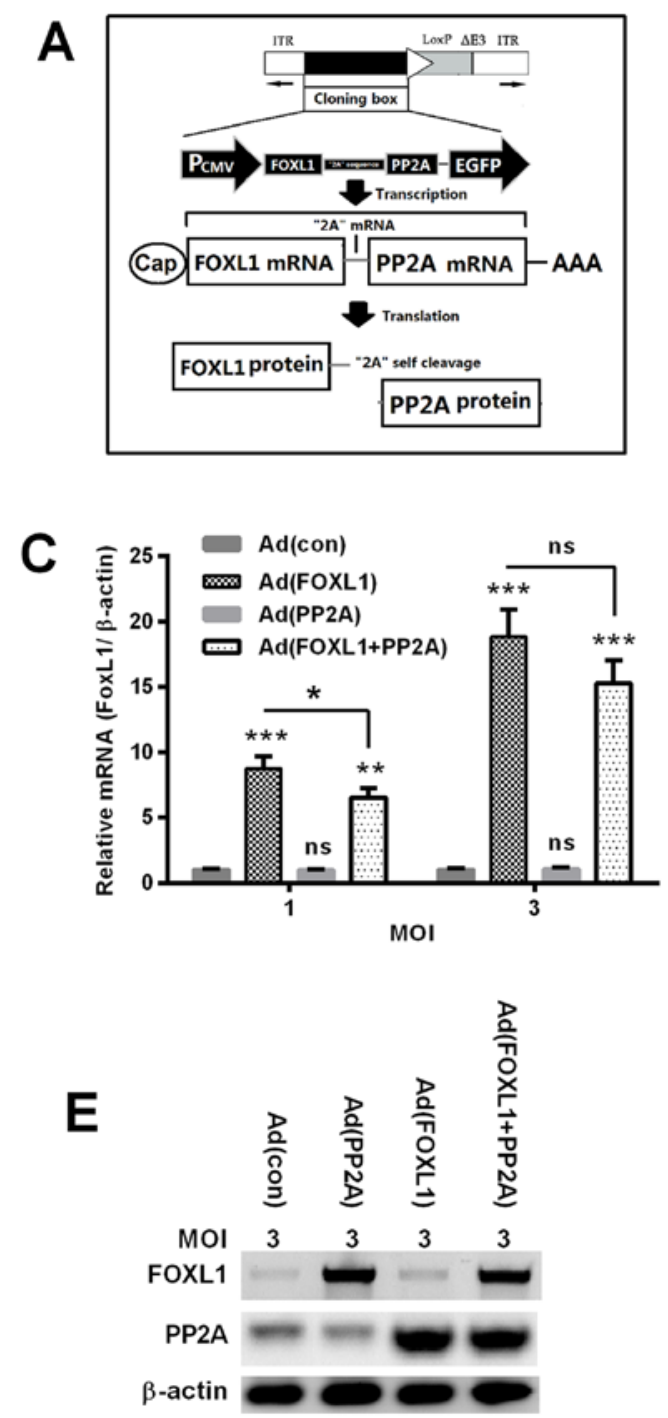

B

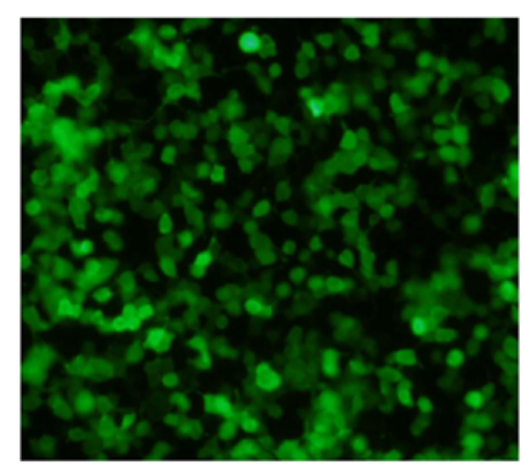

D

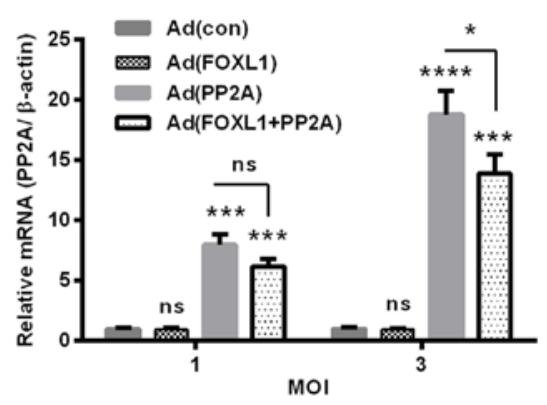

$\mathbf{F}$

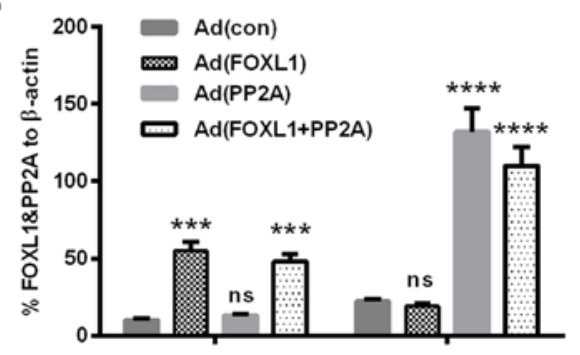

Figure 1. Construction of FOXL1- and PP2A-co-expressed adenovirus. (A) The co-expression strategy of FOXL1 and PP2A with a '2A' self-cleavage sequence in adenovirus. (B) Expression of enhanced green fluorescence protein (EGFP) in Panc-1 cells post-infection with 1 multiplicity of infection (MOI) Ad (con). (C and D) mRNA levels of FOXL1 (C) and PP2A (D) in Panc-1 cells which were infected with 1 or 3 MOI Ad (con), Ad (FOXL1), Ad (PP2A), or Ad (FOXL1 + PP2A) virus for $24 \mathrm{~h}$. (E and F) Western blot analysis of FOXL1 and PP2A in Panc-1 cells infected with 3 MOI Ad (con), Ad (FOXL1), Ad (PP2A), or Ad (FOXL1 + PP2A) virus, with $\beta$-actin as an internal reference protein. ${ }^{*} \mathrm{P}<0.05,{ }^{* *} \mathrm{P}<0.01$, or ${ }^{* * *} \mathrm{P}<0.001$; ns, no significance.

MTT assay. Panc-1 cells were seeded in 96-well plates, at $>85 \%$ confluence, cells were treated with $10 \mu \mathrm{M}$ 5'-FU, and were infected with $3 \mathrm{MOI}$ of Ad (FOXL1), Ad (PP2A), Ad (FOXL1 + PP2A) or Ad (con) respectively, and were incubated for 24 or $48 \mathrm{~h}$. Then, the MTT solution was added and incubated for $4 \mathrm{~h}$. After the MTT solution was aspirated, $100 \mathrm{ml}$ dimethylsulfoxide was added to each well. The absorbance was measured at 570 and $650 \mathrm{~nm}$ using a microplate reader (Bio-Rad Laboratories Inc., Hercules, CA, USA).

Determination of apoptosis and the assay of caspase-3 activity. Apoptosis induced by 5'-FU treatment and adenovirus infection for 24 or $48 \mathrm{~h}$ was determined by Annexin V-FITC kit (Roche Diagnostics, Indianapolis, IN, USA). Cells were incubated in the dark with Annexin V-FITC and PI for $20 \mathrm{~min}$. The apoptotic cells were quantified using flow cytometry, and the percentage of Annexin V-positive cells (early apoptosis) or Annexin V-plus-PI positive cells (late apoptosis) were calculated and were presented as total apoptotic cells. Caspase-3 activity was measured by Apo-ONE homogeneous caspase-3 assay (Promega, Madison, WI, USA) and was calculated and compared with control cells.

Statistical analysis. Quantitative data are presented as the mean \pm standard deviation that is calculated from three independent results. Comparison between two groups was performed with a Student's t-test. A two-way ANOVA test was used for multiple comparisons between three or more groups. $\mathrm{P}<0.05$ was considered to indicate a statistically significant difference.

\section{Results}

Overexpression of FOXL1 and PP2A in panc-1 cells with a FOXL1- and PP2A co-expressed adenovirus. To investigate the tumor suppressive role of FOXL1 and PP2A simultaneously in pancreatic cancer Panc-1 cells, we adopted a strategy to clone the coding sequences of both genes into one opening reading-frame (ORF), and to co-express both 

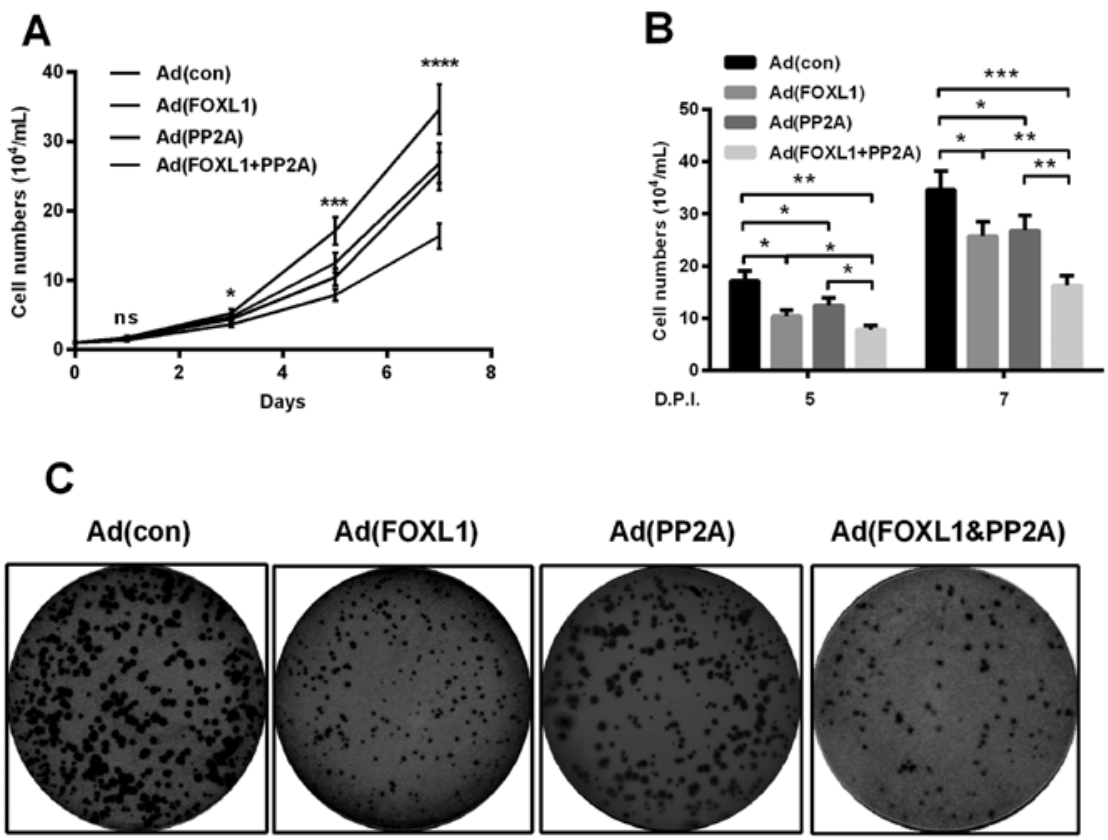

D

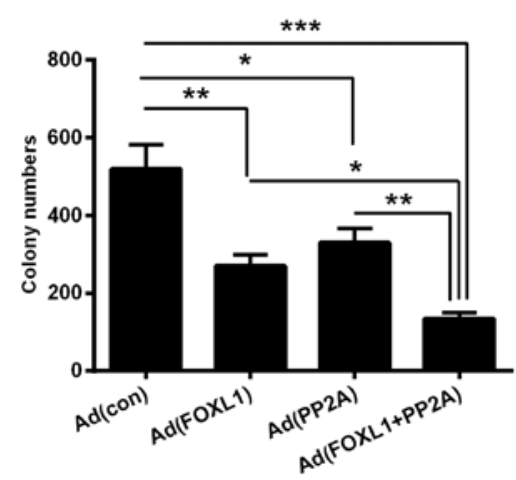

E

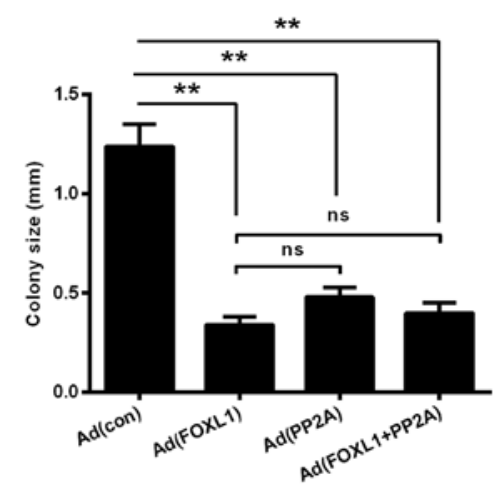

Figure 2. Proliferation of the Panc-1 cells post the overexpression of FOXL1 or (and) PP2A. (A) Proliferation of Panc-1 cells post-infection with 3 MOI Ad (con), Ad (FOXL1), Ad (PP2A), or Ad (FOXL1 + PP2A) virus for 1, 3, 5 or 7 days, with an initial $10^{4}$ cells $/ \mathrm{ml}$ seeded. (B) Difference in the proliferation of Panc-1 cells infected at 3 MOI Ad (con), Ad (FOXL1), Ad (PP2A), or Ad (FOXL1 + PP2A) virus for 5 or 7 days. (C) Colony formation of Panc-1 cells post-infection at 3 MOI Ad (con), Ad (FOXL1), Ad (PP2A), or Ad (FOXL1 + PP2A) virus for 5 days. (D and E) Difference in the number (D) and the size (E) of colonies formed by Panc- 1 cells infected with the above-mentioned virus. ${ }^{*} \mathrm{P}<0.05,{ }^{* *} \mathrm{P}<0.01$, or ${ }^{* * * *} \mathrm{P}<0.001$; ns, no significance.

proteins simultaneously. As shown in Fig. 1A, both FOXL1 and PP2A coding sequences were linked with a self-cleaved ' $2 \mathrm{~A}$ ' peptide coding sequence (32) and were cloned into the multiple cloning sites of the shuttle plasmid, with the promoter of human cytomegalovirus (CMV) immediate early enhancer and promoter $\left(\mathrm{P}_{\mathrm{CMV}}\right)$. The Ad (con) (overexpressing EGFP), Ad (FOXL1) (overexpressing FOXL1), Ad (PP2A) (overexpressing PP2A) or Ad (FOXL1 + PP2A) (overexpressing both FOXL1 and PP2A) was rescued respectively via the co-transfection with the adenoviral genomic plasmid and the shuttle plasmid. Fig. 1B indicates that the infection with the Ad (con) virus caused EGFP expression in $>85 \%$ of Panc- 1 cells. The expression efficiency by the adenovirus of both FOXL1 and PP2A was evaluated in Panc-1 cells post-infection with Ad (FOXL1), Ad (PP2A) or Ad (FOXL1 + PP2A). As shown in Fig. 1C and D, the mRNA level of FOXL1 was significantly promoted by the infection with Ad (FOXL1) or Ad (FOXL1 + PP2A) (either P<0.001 for 1 or $3 \mathrm{MOI})$, and there was no significant difference between Ad (FOXL1) and Ad (FOXL1 + PP2A); Whereas the PP2A mRNA level was significantly upregulated by the infection with Ad (PP2A) or Ad (FOXL1 + PP2A) $(\mathrm{P}<0.001$ or $\mathrm{P}<0.0001)$. Western blotting indicated that the expression of FOXL1 or PP2A was also significantly promoted at protein level in the Panc-1 cells by the infection with Ad (FOXL1)/Ad (FOXL1 + PP2A) virus, or by Ad (PP2A)/Ad $($ FOXL1 + PP2A) virus (Fig. $1 \mathrm{D}-\mathrm{F})(\mathrm{P}<0.001$ or $\mathrm{P}<0.0001)$. Therefore, the three recombinant adenoviruses overexpressed FOXL1 or/and PP2A in Panc-1 cells.

Synergistic inhibition by the co-expression of FOXL1 and PP2A to the proliferation and migration of Panc-1 cells. To investigate the regulatory role of FOXL1 or/and PP2A co-expression on the proliferation of pancreatic cancer cells, the in vitro proliferation of Panc- 1 cells post the infection with Ad (con), Ad (FOXL1), Ad (PP2A) or Ad (FOXL1 + PP2A) 


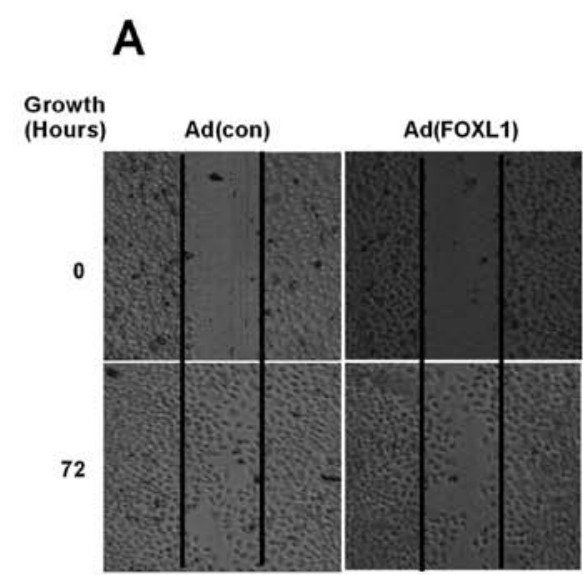

B
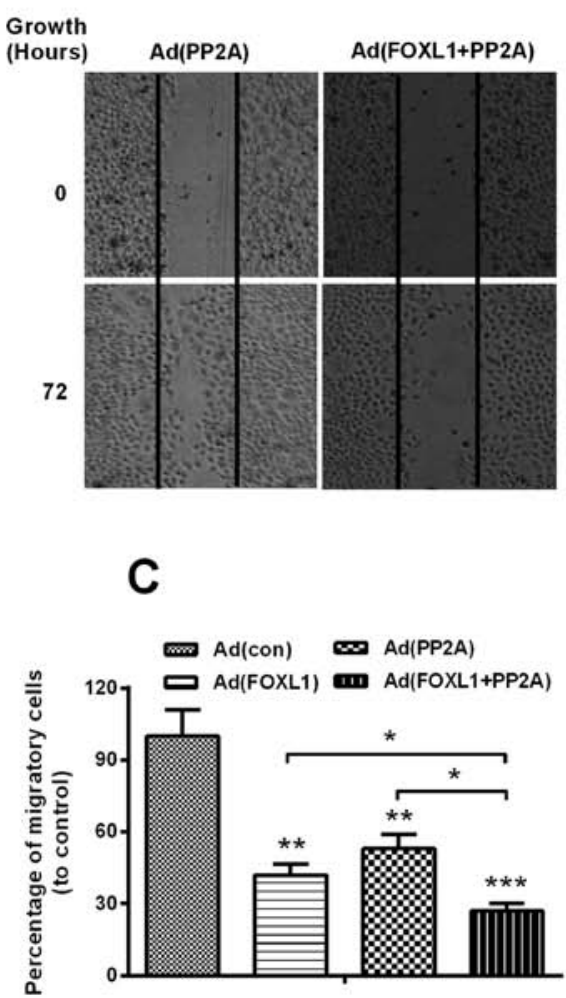

Figure 3. Migration of the Panc-1 cells post-overexpression of FOXL1 or (and) PP2A. (A and B) Wound healing assay of Panc-1 cells, at 0 or $72 \mathrm{~h}$ post-infection at 3 MOI Ad (con) (A), Ad (FOXL1) (A), Ad (PP2A) (B), or Ad (FOXL1 + PP2A) (B) virus. (C) Difference in migratory cell numbers among groups of Ad (con), Ad (FOXL1), Ad (PP2A) and Ad (FOXL1 + PP2A). Statistical significance is shown as ${ }^{*} \mathrm{P}<0.05,{ }^{* *} \mathrm{P}<0.01$, or ${ }^{* * *} \mathrm{P}<0.001$; ns, no significance.

virus was examined with the cell counting and colony formation assays. As shown in Fig. 2A, the growth curve of Panc-1 cells infected with Ad (FOXL1) or with Ad (PP2A) was significantly retardant, compared with the Ad (con) infection. Moreover, the infection with Ad (FOXL1 + PP2A) virus caused a more retardant growth curve of Panc-1 cells $(\mathrm{P}<0.05, \mathrm{P}<0.001$ or $\mathrm{P}<0.0001)$. In particular, the growth efficiency of Panc-1 cells was significant inhibited by the Ad (FOXL1 + PP2A) virus, even compared with the Ad (FOXL1) or Ad (PP2A) virus, at 5- or 7-day post-infection (DPI) $(\mathrm{P}<0.05$ for $5 \mathrm{DPI}$, or $\mathrm{P}<0.01$ for $7 \mathrm{DPI})$.
Then the regulation by the overexpression of FOXL1, PP2A or both molecules on the proliferation of Panc-1 cells was evaluated with the colony forming assay. As indicated in Fig. 2C and D, Panc-1 cells formed less colonies post-infection with Ad (FOXL1), Ad (PP2A) or Ad (FOXL1 + PP2A), compared with the infection with Ad-con $(\mathrm{P}<0.05, \mathrm{P}<0.01$ or $\mathrm{P}<0.001)$. Interestingly, there was also a significant difference in the colony size among the four groups. Colonies in the group of Ad (FOXL1), Ad (PP2A) and Ad (FOXL1 + PP2A) were significantly smaller than in the group of $\mathrm{Ad}$ (con) (Fig. 2E) $(\mathrm{P}<0.01$, respectively) Thus, the co-expression of both FOXL1 and PP2A inhibited the proliferation of Panc-1 cells.

We then determined the regulation of the FOXL1- or/ and PP2A-overexpression on the migration of Panc-1 cells. The migration assay of Panc-1 cells indicated that in contrast to the Ad (con) virus infection, the infection with either Ad (FOXL1), Ad (PP2A) or Ad (FOXL1 + PP2A) at 3 MOI significantly reduced the migration of Panc- 1 cells, there were less migratory cells in the three groups (Fig. 3A-C) $\mathrm{P}<0.01$ for Ad (FOXL1) or Ad (PP2A; P<0.001 for Ad (FOXL1 + PP2A)]. In addition, the migratory cells in the Ad (FOXL1 + PP2A) group were far less than in the Ad (FOXL1) or Ad (PP2A) group ( $\mathrm{P}<0.05$, respectively). Thus, we confirmed the inhibition of the overexpression of FOXL1 in the migration of pancreatic cancer cells.

Co-expression of FOXL1 and PP2A sensitized Panc-1 cells to chemotherapy via enhancing the apoptosis induction. To evaluate the influence of the co-expression of FOXL1 and PP2A on the chemo-sensitivity of pancreatic cancer cells, we also examined the viability reduction and apoptosis induction of Panc-1 cells by $10 \mu \mathrm{M}$ 5'-FU, one of widely-used anticancer agent, post-infection with Ad (con), Ad (FOXL1), Ad (PP2A) or Ad (FOXL1 + PP2A) virus. Fig. 4A indicated that the viability decreased more significantly in the 5'-FU-treated Panc-1 cells, post-infection at 3 MOI Ad (FOXL1), Ad (PP2A) or Ad (FOXL1 + PP2A) virus $[\mathrm{P}<0.05$ for Ad (FOXL1), Ad (PP2A), P<0.01 for Ad (FOXL1 + PP2A)] and the viability reduction was more significant by the infection with Ad (FOXL1 + PP2A) than with Ad (FOXL1), Ad (PP2A) $(\mathrm{P}<0.05$, respectively). The deteriorated apoptosis induction was also confirmed by the overexpression of FOXL1 or/ and PP2A in Panc-1 cells. As shown in Fig. 4B, compared with the Ad (con) infection, there were more apoptotic cells induced by the infection at 3 MOI Ad (FOXL1), Ad (PP2A) or Ad (FOXL1 + PP2A) at $24(\mathrm{P}<0.05$ or $\mathrm{P}<0.01)$ or $48 \mathrm{~h}$ post-infection (HPI) $(\mathrm{P}<0.01$ or $\mathrm{P}<0.001)$, with markedly higher apoptosis in the Ad (FOXL1 + PP2A) group $(\mathrm{P}<0.05$ respectively at $24 \mathrm{HPI}$ or $\mathrm{P}<0.01$ at $48 \mathrm{HPI})$. In addition, we also analyzed the activation and activity of caspase-3, which is the apoptosis-executor, in each groups of cells. Fig.4C-Edemonstrated that there was higher levels of activated caspase-3 (Fig. 4D) and caspase activity (Fig. 4E) induced by the Ad (FOXL1), Ad (PP2A) or Ad (FOXL1 + PP2A) than Ad (con) $(\mathrm{P}<0.05, \mathrm{P}<0.01$ or $\mathrm{P}<0.001$, respectively), particularly higher by the Ad (FOXL1 + PP2A) $(\mathrm{P}<0.05$, respectively). Therefore, we confirmed that the co-expression of FOXL1 and PP2A sensitized Panc-1 cells to 5'-FU via enhancing apoptosis induction. 
A

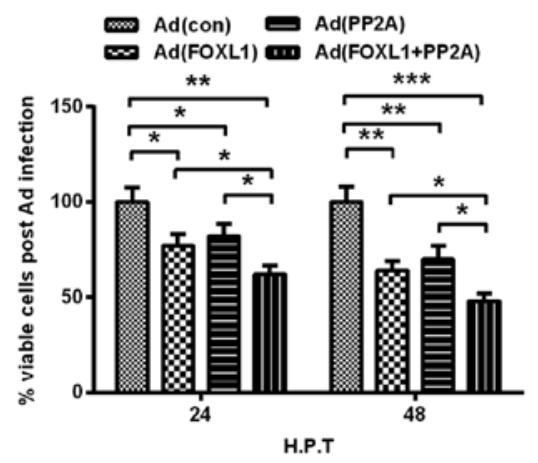

C

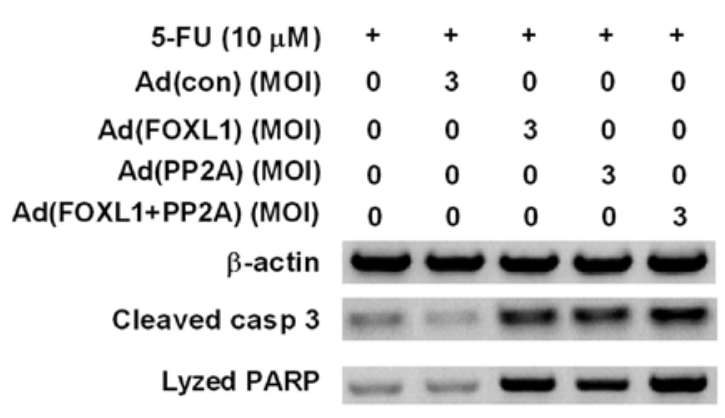

$\mathbf{E}$

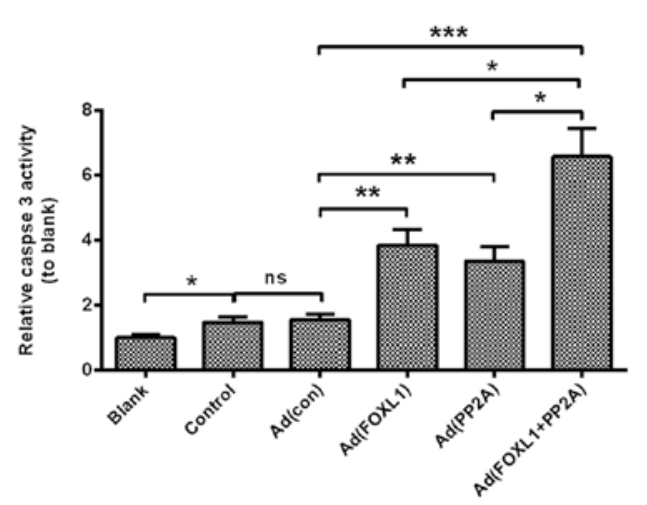

B

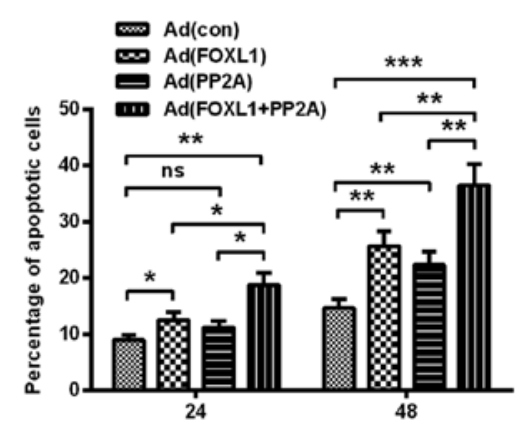

D

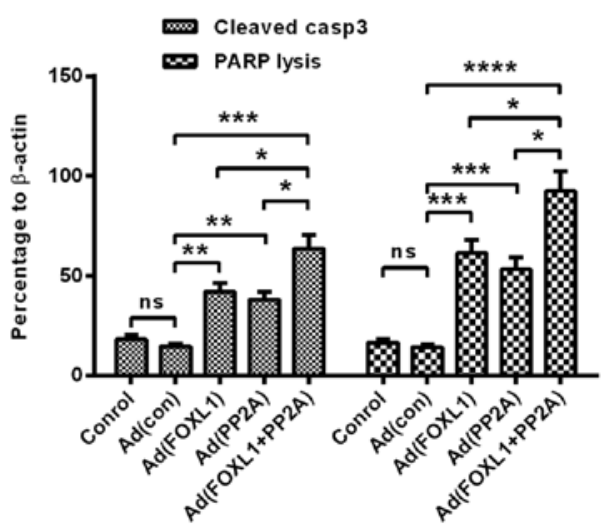

Figure 4. Viability and apoptosis induction in Panc-1 cells post the overexpression of FOXL1 or (and) PP2A, in the presence of $10 \mu \mathrm{M} 5$ '-FU. (A) Relative viability of Panc-1 cells treated with $10 \mu \mathrm{M}$ 5'-FU and infected at 3 MOI Ad (con), Ad (FOXL1), Ad (PP2A) or Ad (FOXL1 + PP2A) virus for 24 or $48 \mathrm{~h}$. (B) Flow cytometric analysis for apoptosis induction by the treatment with $10 \mu \mathrm{M} 5$ '-FU and the infection at 3 MOI adenovirus for 24 or 48 h. (C) Western blot analysis for activated caspase-3 (cleaved casp-3) and its substrate poly ADP ribose polymerase (PARP) in Panc-1 cells post 5'-FU treatment and the adenovirus infection. (D) Relative level of cleaved casp-3 and lyzed PARP in Panc-1 cells post 5'-FU treatment and the adenovirus infection. (E) Relative caspase-3 activity in Panc-1 cells post $5^{\prime}-\mathrm{FU}$ treatment and the adenovirus infection. ${ }^{*} \mathrm{P}<0.05,{ }^{* *} \mathrm{P}<0.01,{ }^{* * * *} \mathrm{P}<0.001$ and ${ }^{* * * * *} \mathrm{P}<0.0001$; ns, no significance.

Co-expression of FOXL1 and PP2A promotes TRAIL, whereas inhibits MYC phosphorylation. TRAIL is a member of the TNF superfamily and triggers apoptosis by recruiting the initiator caspase- 8 and by directly activating downstream effector caspases (33), and FOXL1 has been indicated to inhibit the tumor aggressiveness in human pancreatic cancer via promoting TRAIL (22). The oncogenic MYC (also c-MYC) has also been deregulated in pancreatic cancers and has been confirmed to promote pancreatic cancers (34), and the targeted inhibition of MYC by antagonizing PP2A inhibitor has been indicated to inhibit the growth of breast cancers (35). To explore the possible mechanism in apoptosis induction by the co-expression of FOXL1 and PP2A, we then determined the expression of TRAIL and the phosphorylation of MYC, in Panc-1 cells post-infection at 3 MOI Ad (con), Ad (FOXL1), Ad (PP2A) or Ad (FOXL1 + PP2A) virus. Fig. 5A shows that the mRNA level of TRAIL was significantly upregulated by the infection with Ad (FOXL1) or Ad (FOXL1 + PP2A) for $24 \mathrm{~h}$ (either $\mathrm{P}<0.01)$, rather than the infection with Ad (con) or Ad (PP2A) (no significance). However, the mRNA level of MYC was not significantly regulated by any infection with the above-mentioned virus (Fig. 5B). The western blotting results reconfirmed the regulation of TRAIL and MYC. The protein level of TRAIL was significantly higher in the Panc-1 cells 
A

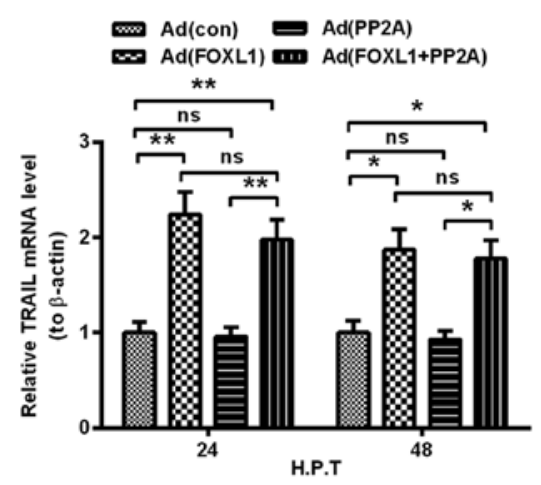

C

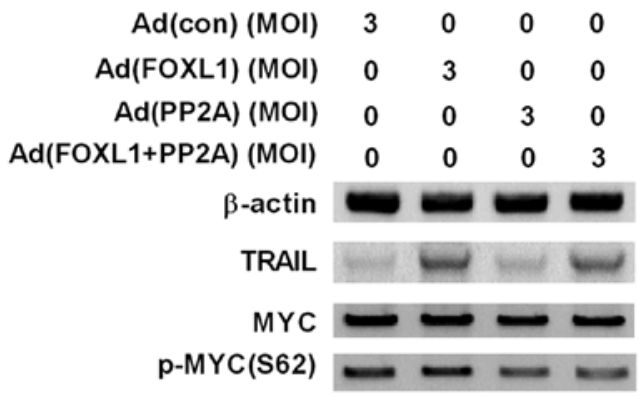

$\mathbf{E}$

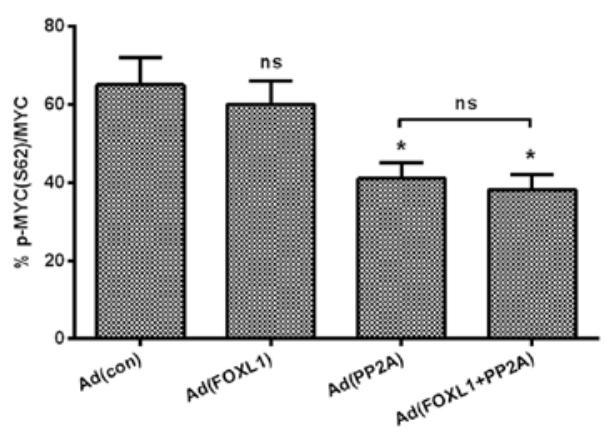

B

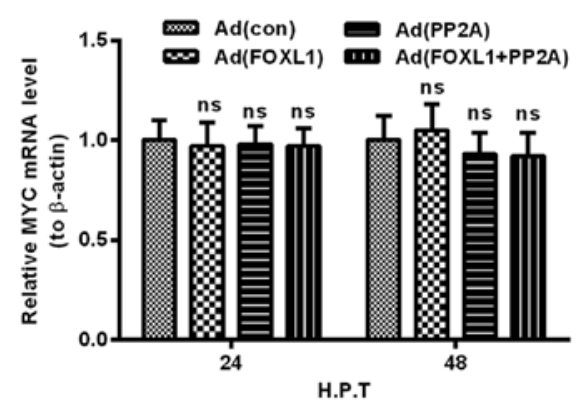

D

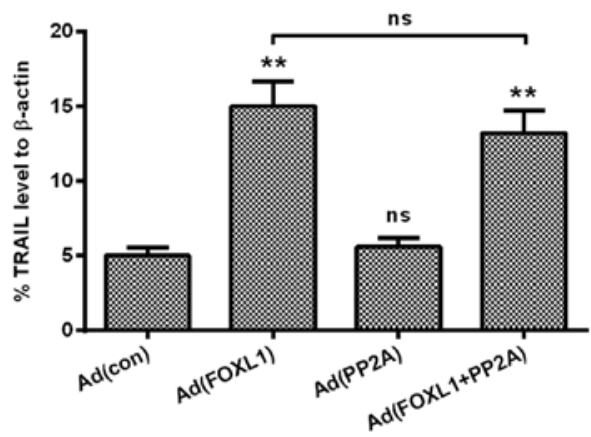

Figure 5. Expression of TRAIL and the phosphorylation of MYC in Panc-1 cells post the co-expression of FOXL1 and PP2A. (A and B) mRNA level of TRAIL (A) or MYC (B) in Panc-1 cells post-infection at 3 MOI Ad (con), Ad (FOXL1), Ad (PP2A), or Ad (FOXL1 + PP2A) virus for 24 h. (C) Western blot analysis of TRAIL, MYC or phosphorylated MYC (Ser at 62) [p-MYC(S62)] in Panc-1 cells post-infection at 3 MOI Ad (con), Ad (FOXL1), Ad (PP2A), or Ad (FOXL1 + PP2A) virus for $48 \mathrm{~h}$. (D and E) Percentage of TRAIL to $\beta$-actin (D) or percent p-MYC(S62) to MYC in each type of infected Panc-1 cells (48 h). Statistical significance is shown as ${ }^{*} \mathrm{P}<0.05$ and ${ }^{* *} \mathrm{P}<0.01 ;$ ns, no significance.

infected with Ad (FOXL1) or Ad (FOXL1 + PP2A) at 3 MOI $(\mathrm{P}<0.01$, respectively), whereas no significantly differene of MYC was found among the groups (Fig. 5C). However, the level of phosphorylated MYC (S62), was significantly downregulated by the infection with either Ad (PP2A) or Ad (FOXL1 + PP2A) (Fig. 5E; P $<0.05$, respectively). Taken together, the co-expression of FOXL1 and PP2A promotes TRAIL, whereas inhibits MYC phosphorylation at S62.

\section{Discussion}

The pathogenesis and the incurable nature of pancreatic cancer, and the rapid metastasis and the poor response to chemo-drugs might contribute to the poor prognosis $(1,36)$. Several pathways have been recognized to regulate the pathogenesis or progression of pancreatic cancers. Hedgehog (Hh) signaling and the nuclear factor- $\mathrm{\kappa B}(\mathrm{NF}-\mathrm{\kappa B})$ pathway have been implicated to involve in the pathogenesis of the disease $(1,37-40)$. Many other pathways have also been found to be deregulated in pancreatic cancers, and to promote the growth aggression of the cancer (36). Such pathways as epidermal growth factor receptor (EGFR) and cyclooxygenase-2 (COX-2) act with an orchestrated interaction each other, and play a significant role in tumorgenesis (40). However, there is no chemo- or immuno-therapeutic agent against these pathways indicating significant effect for PDAC patients. 
Previous studies have indicated the tumor suppressive roles of FOXL1 (22) and PP2A (41) in pancreatic cancers. Thus, the tumor suppression mechanism of FOXL1 and PP2A might facilitate to find novel strategy or target for pancreatic cancer therapy. In the present study, we reconfirmed the tumor suppressive role of either FOXL1 or PP2A in the pancreatic cancer Panc-1 cell line. The Ad-mediated overexpression of either FOXL1 or PP2A significantly inhibited the proliferation of Panc-1 cells via multiple assays, and such overexpression sensitized Panc-1 cells to the treatment with 5'-FU via enhancing apoptosis induction. Moreover, we used a strategy of co-expression of FOXL1 and PP2A to obtain an enhanced tumor suppressive effect on pancreatic cancers. The Ad (FOXL1 + PP2A) virus not only more significantly inhibited the proliferation of Panc-1 cells, but also deteriorated the viability reduction, or enhanced the apoptosis induction in the Panc-1 cells subjected to 5'-FU. 2A peptide is encoded by foot-and-mouth disease virus (FMDV), with a 'self-cleavage' characteristic (42). This 'self-cleavage' peptide composed of $2 \mathrm{~A}$ and $2 \mathrm{~B}$, both of which are translated from one mRNA molecule and function independently (42). Therefore, the $2 \mathrm{~A}$ peptide is well used for the multiple expression of foreign proteins $(43,44)$. In the present study, we confirmed that the adenovirus encoding both FOXL1 and PP2A with the ' $2 \mathrm{~A}$ peptide' linker overexpressed both tumor suppressors in pancreatic cancer cells, and exerted synergistic growth inhibition of pancreatic cancer cells.

TRAIL is a member of the tumor necrosis factor superfamily inducing apoptosis through interaction with the TRAIL-R1 and TRAILR2 receptors (alternatively known as DR4 and DR5, respectively) (45-47). TRAIL has emerged as a potential therapeutic agent due to its selective induction of apoptosis in cancer cells (48). Preliminary clinical trials with TRAIL indicate promising outcomes without obvious toxicity $(49,50)$. The present study presents another confirmation of the antitumor effect of TRAIL via upregulating the upstream FOXL1. Significant promotion of TRAIL in both mRNA and protein levels was confirmed by the infection with either Ad (FOXL1) or Ad (FOXL1 + PP2A). On the contrary, the oncogenic MYC (also namely C-MYC) has been found to be deregulated in pancreatic cancers and has been confirmed to promote pancreatic cancers $(34,51)$. The targeted inhibition of MYC has been indicated to inhibit the growth of breast cancers (35). In mammalian cells, Ser-62 phosphorylation of MYC is associated with the MYC stabilization, and the dephosphorylation of the site by PP2A promotes its polyubiquitination and degradation (52). Previous studies confirmed that inhibited PP2A resulted in increased MYC half-life (53). The current study confirmed the inhibition to Ser-62 phosphorylation of MYC by PP2A overexpression, and it might be associated with the inhibiton of pancreatic cancer cells.

In conclusion, the adenovirus-mediated co-expression of FOXL1 and PP2A with the 2A peptide linker exterts synergistic suppression of pancreatic cancer cells via inhibiting the growth and promoting apoptosis of cancer cells. The coexpressed FOXL1 and PP2A functions independently via upregulating TRAIL (by FOXL1) and reducing the phosphorylation of MYC (by PP2A). Our findings re-confirmed the tumor suppressive role of PP2A and FOXL1 in pancreatic cancer cells, with an enhanced antitumor effect via co-expression of both molecules.

\section{Acknowledgements}

The present study was supported by grants from the First Hospital of China Medical University, Shengyang, China, the Social Development Program from Shenyang and Technology Bureau, China (no. F15-139-9-19), and the Grants-in-aid for Special-Term Professor Scientific Research from the Educational Department of Liaoning Province, China (Liao Zhi Jiao no. 2012-512).

\section{References}

1. Thayer SP, di Magliano MP, Heiser PW, Nielsen CM, Roberts DJ, Lauwers GY, Qi YP, Gysin S, Fernández-del Castillo C, Yajnik V, et al: Hedgehog is an early and late mediator of pancreatic cancer tumorigenesis. Nature 425: 851-856, 2003.

2. Bosetti C, Bertuccio P, Negri E, La Vecchia C, Zeegers MP and Boffetta P: Pancreatic cancer: Overview of descriptive epidemiology. Mol Carcinog 51: 3-13, 2012.

3. Siegel R, Naishadham D and Jemal A: Cancer statistics, 2012. CA Cancer J Clin 62: 10-29, 2012.

4. Kleger A,Perkhofer L and Seufferlein T: Smarter drugs emerging in pancreatic cancer therapy. Ann Oncol 25: 1260-1270, 2014.

5. Jemal A, Siegel R, Xu J and Ward E: Cancer statistics, 2010. CA Cancer J Clin 60: 277-300, 2010.

6. Haberland J, Bertz J, Wolf U, Ziese T and Kurth BM: German cancer statistics 2004. BMC Cancer 10: 52, 2010.

7. Vaccaro V, Sperduti I and Milella M: FOLFIRINOX versus gemcitabine for metastatic pancreatic cancer. N Engl J Med 365: 768-769, 2011.

8. Conroy T, Desseigne F, Ychou M, Bouché O, Guimbaud R, Bécouarn Y, Adenis A, Raoul JL, Gourgou-Bourgade S, de la Fouchardière $\mathrm{C}$, et al; Groupe Tumeurs Digestives of Unicancer; PRODIGE Intergroup: FOLFIRINOX versus gemcitabine for metastatic pancreatic cancer. N Engl J Med 364: 1817-1825, 2011.

9. Moore MJ, Goldstein D, Hamm J, Figer A, Hecht JR, Gallinger S, Au HJ, Murawa P, Walde D, Wolff RA, et al; National Cancer Institute of Canada Clinical Trials Group: Erlotinib plus gemcitabine compared with gemcitabine alone in patients with advanced pancreatic cancer: A phase III trial of the National Cancer Institute of Canada Clinical Trials Group. J Clin Oncol 25: 1960-1966, 2007.

10. Kang SP and Saif MW: Optimal second line treatment options for gemcitabine refractory advanced pancreatic cancer patients. Can we establish standard of care with available data? JOP 9: 83-90, 2008.

11. Bergmann U, Funatomi H, Yokoyama M, Beger HG and Korc M: Insulin-like growth factor I overexpression in human pancreatic cancer: Evidence for autocrine and paracrine roles. Cancer Res 55: 2007-2011, 1995.

12. Rieder S, Michalski CW, Friess H and Kleeff J: Insulin-like growth factor signaling as a therapeutic target in pancreatic cancer. Anticancer Agents Med Chem 11: 427-433, 2011.

13. Seo Y, Baba H, Fukuda T, Takashima M and Sugimachi K: High expression of vascular endothelial growth factor is associated with liver metastasis and a poor prognosis for patients with ductal pancreatic adenocarcinoma. Cancer 88: 2239-2245, 2000.

14. Kindler HL, Niedzwiecki D, Hollis D, Sutherland S, Schrag D, Hurwitz H, Innocenti F, Mulcahy MF, O'Reilly E, Wozniak TF, et al: Gemcitabine plus bevacizumab compared with gemcitabine plus placebo in patients with advanced pancreatic cancer: Phase III trial of the Cancer and Leukemia Group B (CALGB 80303). J Clin Oncol 28: 3617-3622, 2010.

15. Hannenhalli S and Kaestner KH: The evolution of Fox genes and their role in development and disease. Nat Rev Genet 10: 233-240, 2009.

16. Carter ME and Brunet A: FOXO transcription factors. Curr Biol 17: R113-R114, 2007.

17. Paik JH, Kollipara R, Chu G, Ji H, Xiao Y, Ding Z, Miao L, Tothova Z, Horner JW, Carrasco DR, et al: FoxOs are lineage-restricted redundant tumor suppressors and regulate endothelial cell homeostasis. Cell 128: 309-323, 2007. 
18. Tothova Z, Kollipara R, Huntly BJ, Lee BH, Castrillon DH, Cullen DE, McDowell EP, Lazo-Kallanian S, Williams IR, Sears C, et al: FoxOs are critical mediators of hematopoietic stem cell resistance to physiologic oxidative stress. Cell 128: 325-339, 2007.

19. Wang Z, Ahmad A, Li Y, Banerjee S, Kong D and Sarkar FH: Forkhead box M1 transcription factor: A novel target for cancer therapy. Cancer Treat Rev 36: 151-156, 2010.

20. Xia JT, Wang H, Liang LJ, Peng BG, Wu ZF, Chen LZ, Xue L, $\mathrm{Li} \mathrm{Z}$ and $\mathrm{Li} \mathrm{W}$ : Overexpression of FOXM1 is associated with poor prognosis and clinicopathologic stage of pancreatic ductal adenocarcinoma. Pancreas 41: 629-635, 2012.

21. Wang Z, Banerjee S, Kong D, Li Y and Sarkar FH Down-regulation of Forkhead Box M1 transcription factor leads to the inhibition of invasion and angiogenesis of pancreatic cancer cells. Cancer Res 67: 8293-8300, 2007.

22. Zhang G, He P, Gaedcke J, Ghadimi BM, Ried T, Yfantis HG Lee DH, Hanna N, Alexander HR and Hussain SP: FOXL1, a novel candidate tumor suppressor, inhibits tumor aggressiveness and predicts outcome in human pancreatic cancer. Cancer Res 73: 5416-5425, 2013.

23. Arnold HK and Sears RC: A tumor suppressor role for PP2A-B56alpha through negative regulation of c-Myc and other key oncoproteins. Cancer Metastasis Rev 27: 147-158, 2008.

24. Westermarck J and Hahn WC: Multiple pathways regulated by the tumor suppressor PP2A in transformation. Trends Mol Med 14: 152-160, 2008.

25. Eichhorn PJ, Creyghton MP and Bernards R: Protein phosphatase $2 \mathrm{~A}$ regulatory subunits and cancer. Biochim Biophys Acta 1795: 1-15, 2009

26. Farrell AS, Pelz C, Wang X, Daniel CJ, Wang Z, Su Y, Janghorban $\mathrm{M}$, Zhang $\mathrm{X}$, Morgan $\mathrm{C}$, Impey $\mathrm{S}$, et al: Pin1 regulates the dynamics of c-Myc DNA binding to facilitate target gene regulation and oncogenesis. Mol Cell Biol 33: 2930-2949, 2013.

27. Sears RC: The life cycle of c-Myc: From synthesis to degradation. Cell Cycle 3: 1133-1137, 2004

28. Welcker M, Orian A, Grim JE, Eisenman RN and Clurman BE: A nucleolar isoform of the $\mathrm{Fb} 7$ ubiquitin ligase regulates c-Myc and cell size. Curr Biol 14: 1852-1857, 2004.

29. Côme C, Laine A, Chanrion M, Edgren H, Mattila E, Liu X Jonkers J, Ivaska J, Isola J, Darbon JM, et al: CIP2A is associated with human breast cancer aggressivity. Clin Cancer Res 15: 5092-5100, 2009.

30. Wang L, Gu F, Ma N, Zhang L, Bian JM and Cao HY: CIP2A expression is associated with altered expression of epithelial-mesenchymal transition markers and predictive of poor prognosis in pancreatic ductal adenocarcinoma. Tumour Biol 34: 2309-2313, 2013.

31. Schmittgen TD and Livak KJ: Analyzing real-time PCR data by the comparative C(T) method. Nat Protoc 3: 1101-1108, 2008.

32. Szymczak AL, Workman CJ, Wang Y, Vignali KM, Dilioglou S, Vanin EF and Vignali DA: Correction of multi-gene deficiency in vivo using a single 'self-cleaving' $2 \mathrm{~A}$ peptide-based retroviral vector. Nat Biotechnol 22: 589-594, 2004.

33. Ashkenazi A: Targeting death and decoy receptors of the tumour-necrosis factor superfamily. Nat Rev Cancer 2: 420-430 2002.

34. He C, Jiang H, Geng S, Sheng H, Shen X, Zhang X, Zhu S, Chen X, Yang C and Gao H: Expression of c-Myc and Fas correlates with perineural invasion of pancreatic cancer. Int $\mathrm{J}$ Clin Exp Pathol 5: 339-346, 2012.

35. Janghorban M, Farrell AS, Allen-Petersen BL, Pelz C, Daniel CJ, Oddo J, Langer EM, Christensen DJ and Sears RC: Targeting c-MYC by antagonizing PP2A inhibitors in breast cancer. Proc Natl Acad Sci USA 111: 9157-9162, 2014.

36. Sarkar FH, Banerjee S and Li Y: Pancreatic cancer: Pathogenesis, prevention and treatment. Toxicol Appl Pharmacol 224: 326-336, 2007.
37. Berman DM, Karhadkar SS, Maitra A, Montes De Oca R, Gerstenblith MR, Briggs K, Parker AR, Shimada Y, Eshleman JR, Watkins DN, et al: Widespread requirement for Hedgehog ligand stimulation in growth of digestive tract tumours. Nature 425: 846-851, 2003.

38. Liptay S, Weber CK, Ludwig L, Wagner M, Adler G and Schmid RM: Mitogenic and antiapoptotic role of constitutive NF-kappaB/Rel activity in pancreatic cancer. Int J Cancer 105: 735-746, 2003.

39. Karin M: Nuclear factor-kappaB in cancer development and progression. Nature 441: 431-436, 2006.

40. Nakashima H, Nakamura M, Yamaguchi H, Yamanaka N, Akiyoshi T, Koga K, Yamaguchi K, Tsuneyoshi M, Tanaka M and Katano M: Nuclear factor-kappaB contributes to hedgehog signaling pathway activation through sonic hedgehog induction in pancreatic cancer. Cancer Res 66: 7041-7049, 2006.

41. Farrell AS, Allen-Petersen B, Daniel CJ, Wang X, Wang Z, Rodriguez S, Impey S, Oddo J, Vitek MP, Lopez C, et al: Targeting inhibitors of the tumor suppressor PP2A for the treatment of pancreatic cancer. Mol Cancer Res 12: 924-939, 2014.

42. Ryan MD, King AM and Thomas GP: Cleavage of foot-and-mouth disease virus polyprotein is mediated by residues located within a 19 amino acid sequence. J Gen Virol 72: 2727-2732, 1991.

43. Sharma P, Yan F, Doronina VA, Escuin-Ordinas H, Ryan MD and Brown JD: 2A peptides provide distinct solutions to driving stop-carry on translational recoding. Nucleic Acids Res 40: 3143-3151, 2012

44. Szymczak-Workman AL, Vignali KM and Vignali DA: Design and construction of $2 \mathrm{~A}$ peptide-linked multicistronic vectors. Cold Spring Harb Protoc 2012: 199-204, 2012.

45. Pan G, O'Rourke K, Chinnaiyan AM, Gentz R, Ebner R, Ni J and Dixit VM: The receptor for the cytotoxic ligand TRAIL. Science 276: 111-113, 1997.

46. Walczak H, Degli-Esposti MA, Johnson RS, Smolak PJ, Waugh JY, Boiani N, Timour MS, Gerhart MJ, Schooley KA, Smith CA, et al: TRAIL-R2: A novel apoptosis-mediating receptor for TRAIL. EMBO J 16: 5386-5397, 1997.

47. Sheridan JP, Marsters SA, Pitti RM, Gurney A, Skubatch M, Baldwin D, Ramakrishnan L, Gray CL, Baker K, Wood WI, et al: Control of TRAIL-induced apoptosis by a family of signaling and decoy receptors. Science 277: 818-821, 1997.

48. Walczak H, Miller RE, Ariail K, Gliniak B, Griffith TS, Kubin M, Chin W, Jones J, Woodward A, Le T, et al: Tumoricidal activity of tumor necrosis factor-related apoptosis-inducing ligand in vivo. Nat Med 5: 157-163, 1999.

49. Newsom-Davis T, Prieske S and Walczak H: Is TRAIL the holy grail of cancer therapy? Apoptosis 14: 607-623, 2009.

50. Mahalingam D, Szegezdi E, Keane M, de Jong S and Samali A: TRAIL receptor signalling and modulation: Are we on the right TRAIL? Cancer Treat Rev 35: 280-288, 2009.

51. Schild C, Wirth M, Reichert M, Schmid RM, Saur D and Schneider G: PI3K signaling maintains c-myc expression to regulate transcription of E2F1 in pancreatic cancer cells. Mol Carcinog 48: 1149-1158, 2009.

52. Escamilla-Powers JR and Sears RC: A conserved pathway that controls c-Myc protein stability through opposing phosphorylation events occurs in yeast. J Biol Chem 282: 5432-5442, 2007.

53. Yeh E, Cunningham M, Arnold H, Chasse D, Monteith T, Ivaldi G, Hahn WC, Stukenberg PT, Shenolikar S, Uchida T, et al: A signalling pathway controlling c-Myc degradation that impacts oncogenic transformation of human cells. Nat Cell Biol 6 : 308-318, 2004. 